\title{
A Quantum Model for the Dynamics of Cold Dark Matter
}

\author{
Tim Zimmermann ${ }^{1}\left(\mathbb{D}\right.$, Massimo Pietroni $^{2,3}{ }^{\mathbb{D}}$, Javier Madroñero ${ }^{4}\left(\mathbb{D}\right.$, Luca Amendola ${ }^{1}(\mathbb{D})$ and \\ Sandro Wimberger $2,5, *$ (D) \\ 1 ITP, Heidelberg University, Philosophenweg 16, 69120 Heidelberg, Germany; \\ zimmermann@thphys.uni-heidelberg.de (T.Z.); l.amendola@thphys.uni-heidelberg.de (L.A.) \\ 2 Dipartimento di Scienze Matematiche, Fisiche e Informatiche, Università di Parma, Campus Universitario, \\ Parco Area delle Scienze n. 7/a, 43124 Parma, Italy; massimo.pietroni@unipr.it \\ 3 INFN, Sezione di Padova, 35131 Padova, Italy \\ 4 Centre for Bioinformatics and Photonics (CiBioFi), Universidad del Valle, A.A. 25360, 760032 Cali, Colombia; \\ javier.madronero@correounivalle.edu.co \\ 5 INFN, Sezione di Milano Bicocca, Gruppo Collegato di Parma, 43124 Parma, Italy \\ * Correspondence: sandromarcel.wimberger@unipr.it; Tel.: +39-0521-90-5213
}

Received: 24 October 2019; Accepted: 8 November 2019; Published: 13 November 2019

check for updates

\begin{abstract}
A model for cold dark matter is given by the solution of a coupled Schrödinger-Poisson equation system. We present a numerical scheme for integrating these equations, discussing the problems arising from their nonlinear and nonlocal character. After introducing and testing our numerical approach, we illustrate key features of the system by numerical examples in $1+1$ dimensions. In particular, we study the properties of asymptotic states to which the numerical solutions converge for artificial initial conditions.
\end{abstract}

Keywords: nonlinear Schrödinger equation; Gross-Pitaevskii equation; Schrödinger-Poisson equation; Bose-Einstein condensate; dark matter

\section{Introduction}

Nonlinear equations are ubiquitous in physics. In optics [1], they serve as back reaction models of the media onto the propagating beam. In many-body systems, mutual interactions give rise to effective nonlinear potentials within the mean field approximation [2,3]. Especially intriguing are nonlocal nonlinear problems arising, for instance, from long-range forces such as gravity. In this paper we study a quantum dynamical model for dark matter which is of recent interest in cosmology, see [4-8], as it recovers known features of dynamically cold dark matter (CDM) on sufficiently large spatial scales while alleviating shortcomings of the classical description on small scales due to the quantum nature of the model.

The rich set of hierarchical structures we observe in the universe, ranging from kiloparsec galaxies up to the cosmic web visible on gigaparsec scales, is understood as an evolution snapshot of all gravitationally interacting matter starting from dynamically cold initial conditions [9]. Observational data [10] suggests that the main driver of this large-scale structure formation process is dark matter, a non-baryonic matter component of yet undetermined character and origin that only interacts gravitationally. The full fledged dynamics of CDM, i.e., the evolution of its probability distribution in phase space is often modeled by means of a Boltzmann equation. Since the expected number of interacting particles is immense, relaxation times are extremely large and thus one considers structure formation only as collisionless problem [11]. A brief overview of the classical CDM description is given in Section 1.1. 
An alternative approach in modeling large scale structure formation, first suggested in this context by [12], is to model the temporal evolution of dark matter as a complex scalar field $\psi(x, t)$ governed by Schrödinger's equation with a nonlocal, nonlinear self-interaction potential given as the solution to Poisson's field equation. Depending on the point of view, one can either interpret this model as a distinct description of dark matter known as fuzzy dark matter [13], in which structure formation is driven by a cosmic Bose-Einstein condensate trapped in its own gravitational potential and where the expansion of space sets the time dependent interaction strength; or as a field theoretical approximation to the classical Boltzmann description [14-17]. A more detailed discussion about both the Bose-Einstein condensate and the Boltzmann-Schrödinger correspondence is given in Section 1.2.

We note in passing that the application of the Schrödinger-Poisson model as a nonlinear equation system can also be found in other fields, such as fundamental quantum mechanics, see e.g., [18], and nonlinear optics [19]. In the former it acts as model for quantum collapse theory. The latter studies light propagation in nonlinear media in which steady state heat transfer due to the absorption of light along the light path induces a change in the refractive index which is formally identical to the type of nonlocal nonlinearity we encounter in our gravitational problem.

\subsection{Classical Description of Cold Dark Matter}

A theoretical description of the temporal evolution of CDM can be developed in the framework of classical Hamiltonian mechanics yielding a set of coupled differential equations known as the Vlasov-Poisson or collisionless Boltzmann equation which in flat space reads:

$$
\begin{aligned}
0 & =\frac{\partial f}{\partial t}+\frac{u}{a^{2}} \cdot \nabla_{x} f-\nabla_{x} V \cdot \nabla_{p} f \\
\triangle V & =\frac{4 \pi G \rho_{m 0}}{a}\left(\frac{\int \mathrm{d}^{3} u f(x, u, t)}{\left\langle\int \mathrm{d}^{3} u f(\boldsymbol{x}, \boldsymbol{u}, t)\right\rangle_{\mathrm{Vol}}}-1\right) .
\end{aligned}
$$

In this description, dark matter is modeled as the smooth probability density $f(\boldsymbol{x}, \boldsymbol{u}, t)$ trapped in its own gravitational potential and evolving in phase space spanned by comoving position $x$ and conjugate velocity $\boldsymbol{u}$. The latter quantities factor out the cosmological expansion of space as measured by the dimensionless scale factor $a(t) . a(t)$ depends on the cosmological model, in particular on the present day dimensionless matter density parameter $\Omega_{m 0}$ and dark energy density $\Omega_{\Lambda 0}$. Radiation contributions to the total energy budget can typically be neglected. Thus, we restrict ourselves to models with $1=\Omega_{m 0}+\Omega_{\Lambda 0}$. Further details are given in Appendix A. Integrating $f$ over velocity space yields the dark matter particle density $n(x, t)$ that consists of a spatially homogeneous background contribution and a density fluctuation associated with the peculiar motion of the particles due to gravity. Hence, the bracket term in Equation (1) measures the relative excess density with respect to the background. It is called density contrast $\delta(x, t)$. Both the analytical and numerical treatment of Equation (1) is challenging due its nonlocal nonlinearity and the large number of degrees of freedom in $f$. Hence, simplifications are in order.

Analytically, one often resorts to a hydrodynamical model of Equation (1) by integrating over velocity or position space to obtain equations of motion for the marginal distributions of $f$. Assuming a dynamically cold distribution with vanishing velocity dispersion of the form of a phase space sheet, one obtains:

$$
f(x, u, t)=n(x, t) \delta_{D}\left(\boldsymbol{u}-\nabla_{x} \phi_{u}(x, t)\right),
$$

where $\phi_{u}$ denotes the potential of the irrotational velocity flow. Although simple in its formulation, this model breaks down at shell crossing, the moment in the evolution of Equation (2) when the initial phase space sheet becomes perpendicular to the spatial axis. This so called dust model is incapable of describing multiple matter streams or virialized matter structures as we expect them.

Sampling the true distribution function by means of Newtonian test particles and only following their motion and mutual interaction gives rise to $N$-Body simulations $[20,21]$ which proved to be an 
invaluable tool in cosmology. Although successful in predicting the correct large scale features of the cosmic matter distribution, $\mathrm{N}$-Body simulations produce density profiles of collapsed structures with cuspy core regions which are not supported by observational data. Whether this cusp-core problem is a prediction of the CDM model or an artifact of $N$-Body simulations lacking crucial physical effects (e.g., baryonic feedback) is still open to debate. We refer to [22] for an in depth review of the cusp-core problem as well as other puzzling phenomena collectively often referred to as "small scale crisis".

\subsection{The Schrödinger-Poisson Model (SPM)}

An alternative approach in modeling large scale structure formation, first suggested by [12], is to model the temporal evolution of dark matter as a complex scalar field $\psi(x, t)$ governed by Schrödinger's equation with a nonlocal and nonlinear self-interaction potential given as the solution to Poisson's field equation:

$$
\begin{aligned}
i\left(\frac{\hbar}{m}\right) \partial_{t} \psi(x, t) & =\left[-\left(\frac{\hbar}{m}\right)^{2} \frac{1}{2 a^{2}(t)} \Delta+V(x, t)\right] \psi(x, t), \\
\triangle V & =\frac{4 \pi G \rho_{m 0}}{a(t)}\left(\frac{|\psi(x, t)|^{2}}{\left\langle|\psi(x, t)|^{2}\right\rangle_{\mathrm{Vol}}}-1\right) .
\end{aligned}
$$

Here the potential is already divided by the particle mass. We briefly remark on the different physical interpretations of Equation (3) that exist in the literature: One may regard Equation (3) as an alternative approximation to Equation (1), numerically compared to $\mathrm{N}$-Body simulations and analytically competing against the dust-model, see e.g., [14-17]. Here, both wavefunction and $\mu=\frac{\hbar}{m}$ do not carry any quantum mechanical meaning and are best understood as a classical complex field and a free model parameter. In the semiclassical limit, $\mu \rightarrow 0$, we expect good correspondence between observables arising from Equations (1) and (3), respectively. For instance, the numerical study in [16] suggests convergence of the wavefunction's gravitational potential to the classical result of Equation (1) as $\mu^{2}$.

In fact, if Equation (3) is augmented with Husimi's quasi probability distribution,

$$
f_{H}(\boldsymbol{x}, \boldsymbol{u}, t)=\frac{1}{\left(2 \pi \sigma_{x} \sigma_{u}\right)^{3}} \int \mathrm{d}^{3} x^{\prime} \mathrm{d}^{3} u^{\prime} e^{-\frac{\left(x-x^{\prime}\right)^{2}}{2 \sigma_{x}^{2}}} e^{-\frac{\left(u-u^{\prime}\right)^{2}}{2 \sigma_{u}^{2}}} f_{W}\left(\boldsymbol{x}^{\prime}, \boldsymbol{u}^{\prime}, t\right) \quad \text { with } \sigma_{x} \sigma_{u}=\frac{\mu}{2},
$$

a smoothed version of Wigner's distribution,

$$
f_{W}(x, u, t)=\frac{1}{(2 \pi \mu)^{3}} \int \mathrm{d}^{3} x^{\prime} \psi^{*}\left(x+\frac{x^{\prime}}{2}\right) \psi\left(x-\frac{x^{\prime}}{2}\right) e^{\frac{i u \cdot x^{\prime}}{\mu}} .
$$

It was shown in [14] that the phase space distribution $f_{H}$ associated with the dynamics of the scalar field $\psi$ approximates the dynamics of the smoothed Vlasov distribution $\bar{f}$, see Equation (4), in the following sense:

$$
\partial_{t}\left(f_{H}-\bar{f}\right)=\mathcal{O}\left(\mu^{2}\right) .
$$

Moreover, higher order moments such as the velocity dispersion are non-vanishing and can be readily computed with only the knowledge of the wavefunction. The non-vanishing hierarchy of distribution moments induces a rich set of phenomena such as shell crossings, multi-streaming, and relaxation into the equilibrium state that can all be studied within the SPM $[14,15,17]$. Section 3.1 illustrates these prototypical evolution stages by studying the gravitational collapse of a sinusoidal density perturbation in phase space by means of Husimi's distribution, Equation (4).

Alternatively, one can interpret Equation (3) as a distinct model for dark matter competing against the established CDM paradigm. In this fuzzy dark matter picture [13], Equation (3) arises as the nonrelativistic weak field limit of the Klein-Gordon-Einstein equation. This equation describes 
ultralight scalar bosons-so called axions-that constitute a cosmic Bose-Einstein condensate trapped in its own gravitational potential. The boson mass is expected to be of the order of $m \approx 10^{-22} \mathrm{eV}$ and, in fact, state of the art $3+1$ dimensional simulations of axion dark matter [6] set $m=8 \times 10^{-23} \mathrm{eV}$ by fitting simulated halo density profiles against observational data. The remarkable feature of this model is that due to the minuscule axion mass the de-Broglie wavelength takes on macroscopic kiloparsec values such that Heisenberg's uncertainty principle acts on cosmic scales [23] and washes out the small scale structure that the CDM paradigm struggles to model correctly. To see the regularizing nature of fuzzy dark matter at small scales one can examine its associated fluid description. Using the Madelung transformation [24],

$$
\psi(x, t)=\sqrt{n(x, t)} \exp \left(i \frac{\phi_{u}(x, t)}{\mu}\right),
$$

as ansatz for the wavefunction, yields a modified Euler equation for the velocity field $u=\nabla \phi_{u}$ :

$$
0=\partial_{t} \boldsymbol{u}+\frac{1}{a^{2}}\left(\boldsymbol{u} \cdot \nabla_{x}\right) \boldsymbol{u}+\nabla_{x} V-\frac{\mu^{2}}{2 a^{2}} \nabla\left(\frac{\triangle \sqrt{n}}{\sqrt{n}}\right) .
$$

This equation contains an additional pressure term compared to the dust model, see discussion around Equation (2), that counteracts the gravitational collapse. More specifically, in $3+1$ dimensions each collapsed and virialized matter structure contains a flat solitonic core embedded in a dark matter halo of universal shape, known as the Navarro-Frenk-White (NFW) profile [25]. We refer to [6,23] for cosmological simulations in $3+1$ dimensions and refer to $[4,5,7,8]$ for numerical studies carried out in a static spacetime.

The present work focuses on a systematic analysis of the $1+1$ case of Equation (3). The paper is structured as follows: In Section 2 we provide details of the numerical procedure used to integrate Equation (3) in time as well as a convergence study for synthetic initial conditions. Section 3 then focuses on recovering key characteristics of the fuzzy dark matter model known from $3+1$ dimensions in only one spatial dimension: Section 3.1 investigates the phase space evolution of spatially non-localized initial conditions in an expanding spacetime by means of Husimi's distribution, Equation (4). To assess both qualitative and quantitative features of the dynamical equilibrium state Section 3.2 specializes to spatially localized initial conditions. In particular, Section 3.2.1 illustrates the existence of flat density cores. Contrary to the $3+1$ case, the core region is not stationary but oscillates in time, which we interpret as a nonlinear superposition of a solitonic ground state and higher order nonlinear modes also known as breathers [26]. Finally, Section 3.2.2 investigates the halo of a collapsed structure in static spacetime and recovers the classical CDM expectation of a matter-density obeying a power-law scaling combined with a distinct cutoff radius, see e.g., [27,28]. We conclude in Section 4 .

\section{Numerical Approach}

Although structure formation is naturally studied in $3+1$ dimensions, we will restrict ourselves to only one spatial dimension. Two reasons motivate this decision: Firstly, from a physical point of view the one dimensional problem is an intriguing system in its own right with its own special properties. Moreover, as we will see, important qualitative aspects of the full fledged $3+1$ phenomenology can already be understood in $1+1$ dimensions. Secondly, integrating Equation (3) is a computationally involved problem especially due to the fact that vastly different spatial scales-solitonic cores with radii of $\sim 100$ parsec up to megaparsec filaments [6], four orders of magnitude-need to be resolved. This led [6] to using a sophisticated adaptive mesh refinement, a technique out of the scope of this work. Setting the spatial resolution aside, it is generally difficult to find any reliable convergence analysis in the literature, particularly for dynamical background models. Hence, it is only natural to start with the simplest system and gradually increase its complexity once all aspects of both the model and the numerical method are reliably understood. 
That being said, let us specialize Equation (3) to $1+1$ dimensions. We follow the convention of $[6,29,30]$ and define a dimensionless spatial coordinate $x^{\prime}$ as well as an adimensional time parameter $t^{\prime}$ by:

$$
x^{\prime} \equiv \frac{1}{\mu^{\frac{1}{2}}}\left[\frac{3}{2} H_{0}^{2} \Omega_{m 0}\right]^{\frac{1}{4}} x, \quad \mathrm{~d} t^{\prime} \equiv \frac{1}{a^{2}}\left[\frac{3}{2} H_{0}^{2} \Omega_{m 0}\right]^{\frac{1}{2}} \mathrm{~d} t .
$$

$H_{0}, \Omega_{m 0}$ denote the present day Hubble constant and matter density parameter respectively. Then the potential becomes $V^{\prime} \equiv \frac{a}{\mu}\left[\frac{3}{2} H_{0}^{2} \Omega_{m 0}\right]^{-\frac{1}{2}} V$. Dropping primes for all dimensionless quantities and imposing periodic boundary conditions brings Equation (3) into the numerically more convenient form:

$$
\begin{array}{rlrl}
i \partial_{t} \Psi(x, t) & =\left[-\frac{1}{2} \partial_{x}^{2}+a(t) V\left[|\Psi|^{2}\right]\right] \Psi(x, t), & x \in \Omega=\left[-\frac{L}{2}, \frac{L}{2}\right), \\
\partial_{x}^{2} V & =|\Psi(x, t)|^{2}-1, & \\
\Psi(0, t) & =\Psi(L, t), & & \text { boundary conditions, } \\
V(0, t) & =V(L, t)=0, & \text { initial condition. }
\end{array}
$$

where $L$ is the box length in dimensionless units. The scale factor $a(t)$ acts as time-dependent coupling strength for the dimensionless potential $V$. The adimensional wavefunction $\Psi$ is defined such that its norm square measures the comoving number density relative to the constant homogeneous background,

$$
|\Psi(x, t)|^{2} \equiv \frac{n(x, t)}{n_{m 0}}, \quad \int_{\Omega} \mathrm{d} x|\Psi|^{2}=L
$$

making $\langle\delta\rangle=0$ manifest.

By this convention, the effective Planck constant $\mu=\frac{\hbar}{m}$ only enters the equations as part of the spatial scale and energy scale in which we choose to measure physical quantities. Therefore, solving Equation (10) for a fixed value of the adimensional domain length and initial conditions satisfying $\langle\delta\rangle=0$ yields the correct dynamics to all physical domains for which $L / \sqrt{\mu}$ is constant.

\subsection{Numerical Method}

Equation (10) is a non-autonomous Schrödinger equation with a nonlocal and nonlinear interaction potential. The nonlocal behavior becomes apparent if we formally solve Poisson's equation by means of a convolution integral. Let $G\left(x, x^{\prime}\right)$ be Green's function. Then:

$$
i \partial_{t} \Psi(x, t)=-\frac{1}{2} \partial_{x}^{2} \Psi(x, t)+a(t)\left[\int_{\Omega} \mathrm{d} x^{\prime} G\left(x, x^{\prime}\right)\left(|\Psi|^{2}\left(x^{\prime}, t\right)-1\right)\right] \Psi(x, t) .
$$

The autonomous-static cosmology — case is a common numerical problem arising in nonlinear optics, e.g., [19,31], and a multitude of methods for solving exist in the literature. We refer to [32] for a review of common numerical methods involving nonlinear Schrödinger/Gross-Pitaevskii equations.

For sufficiently smooth initial conditions, one often resorts to symmetric operator splittings combined with fast Fourier transformations (FFT) to diagonalize the momentum operator and cast Poisson's equation into an algebraic form. The advantage of this approach lies in its ease of use, norm and energy preserving property and satisfactory accuracy at moderate computational cost. This needs to be contrasted with implicit schemes which eventually have to solve (multiple) linear or even nonlinear sets of equations per timestep. Moreover, rigorous error and stability bounds exist for second and higher order splittings that only depend on the regularity of the initial conditions $[33,34]$. 
The non-autonomous, time-dependent case requires special treatment since the commutator $\left[a\left(t_{1}\right) V, a\left(t_{2}\right) V\right] \neq 0$. Hence, time ordering becomes an issue in the Poisson part, see Equation (16) below. In the more general context of evolutionary equations [35], uses a truncation of the Magnus expansion to construct a time averaged problem that can subsequently be solved with optimized splitting methods, see e.g., [36]. In the present work, we employ a second order Strang splitting on the time averaged problem, which can be understood as the simplest representative of the class of splittings developed in [35]. To this end, we consider

$$
\begin{aligned}
& i \partial_{t} \Psi_{A}(x, t)=-\frac{1}{2} \partial_{x}^{2} \Psi_{A}(x, t) \quad \text { and } \\
& i \partial_{t} \Psi_{B}(x, t)=a(t) V\left[\left|\Psi_{B}\right|^{2}\right] \Psi_{B}(x, t), \quad \partial_{x}^{2} V=\left|\Psi_{B}(x, t)\right|^{2}-1
\end{aligned}
$$

as independent problems. Ideally, we would like to integrate Equations (15) and (16) exactly to obtain the time evolution operator $U_{A / B}(t, \Delta t) \Psi_{A / B}=\Psi_{A / B}(t+\Delta t)$. This is readily done for the kinetic (A) problem. If $\mathcal{F}$ denotes the Fourier transform, we have:

$$
U_{A}(\Delta t) \Psi_{A}=\mathcal{F}^{-1} e^{-\frac{i}{2} \Delta t k^{2}} \mathcal{F} \Psi_{A}
$$

The potential problem (B) needs an additional approximation due to its explicit time dependence entering by the scale factor. Therefore, we consider the Magnus expansion [37], of the time evolution operator:

$$
U_{B}(t, \Delta t) \Psi_{B}=\exp \left(-i \sum_{k=1}^{\infty} \Omega_{k}\right) \Psi_{B}
$$

If we're only interested in second order accuracy in time, it suffices to truncate the series after the first term to arrive at:

$$
U_{B}(t, \Delta t) \Psi_{B}=\exp \left(-i \int_{t}^{t+\Delta t} \mathrm{~d} t^{\prime} a\left(t^{\prime}\right) V\left[\left|\Psi_{B}\left(x, t^{\prime}\right)\right|^{2}\right]\right) \Psi_{B}+\mathcal{O}\left(\Delta t^{3}\right) .
$$

Since the potential $V$ is real, subproblem B conserves the wavefunction's squared norm,

$$
\partial_{t}\left(\left|\Psi_{B}\right|^{2}\right)=\partial_{t}\left(\Psi_{B}^{*}\right) \Psi_{B}+\partial_{t}\left(\Psi_{B}\right) \Psi_{B}^{*}=\left[i a(t) V\left[\left|\Psi_{B}(x, t)\right|^{2}\right]-i a(t) V\left[\left|\Psi_{B}(x, t)\right|^{2}\right]\right]\left|\Psi_{B}\right|^{2}=0,
$$

and therefore any function of $\left|\Psi_{B}\right|^{2}$ is an integral of motion. This reduces Equation (19) to:

$$
U_{B}(t, \Delta t) \Psi_{B}=\exp \left(-i V\left[\left|\Psi_{B}(x, t)\right|^{2}\right] \int_{t}^{t+\Delta t} \mathrm{~d} t^{\prime} a\left(t^{\prime}\right)\right) \Psi_{B}+\mathcal{O}\left(\Delta t^{3}\right)
$$

It is in general not possible to integrate the scale factor $a(t)$ as a function of our time parameter in Equation (9) in closed form for arbitrary values of $\Omega_{m 0}$, see Appendix A. Hence, we use a trivial quadrature rule, the midpoint method, consistent with the truncation introduced in Equation (19) to arrive at:

$$
U_{B}(t, \Delta t) \Psi_{B}=\exp \left(-i \Delta t a\left(t+\frac{\Delta t}{2}\right) V\left[\left|\Psi_{B}(x, t)\right|^{2}\right]\right) \Psi_{B}+\mathcal{O}\left(\Delta t^{3}\right)
$$

An approximation for the time evolution operator of the combined problem, Equation (10), is obtained by a symmetric splitting of the form:

$$
\Psi(t+\Delta t)=U_{A+B}(t, \Delta t) \Psi(t)=U_{A}\left(\frac{\Delta t}{2}\right) U_{B}(t, \Delta t) U_{A}\left(\frac{\Delta t}{2}\right) \Psi(t)+\mathcal{O}\left(\Delta t^{3}\right)
$$


To arrive at a full discretization, we choose a uniform spatial grid of $N$ points separated by $\Delta x=L / N$ and only store both the wavefunction and the potential at discrete grid sites. The latter is easily computed by yet another Fourier transformation:

$$
V\left[|\Psi|^{2}\right]=\mathcal{F}^{-1}\left(\frac{-1}{k^{2}} \mathcal{F}\left(\left|\Psi_{B}\right|^{2}-1\right)\right)
$$

Since $\langle\delta\rangle=\left\langle|\Psi|^{2}-1\right\rangle=0$ by statistical homogeneity-a consequence of the cosmological principle [38] - no singularity is found at $k=0$. The presented scheme has the advantages that it is explicit, unitary and for $a=$ const. energy preserving. Moreover, for $a=$ const., it reduces to a standard split-step Fourier method as it was also used, e.g., in [8], for collapse studies in static background cosmologies. The computational cost is dominated by four FFTs per timestep and is consequently $\mathcal{O}(N \log N)$ in time and $\mathcal{O}(N)$ in memory, where $\mathrm{N}$ is the grid dimension.

\subsection{Convergence Study}

To check the convergence of the employed scheme we consider a dimensionless box of size $L=100$ and $N=16,384$ grid points, see Equation (9), and place a sinusoidal perturbation of the homogeneous background density into the box as initial state:

$$
\Psi_{0}(x)=\sqrt{\delta_{0}(x)+1}=\sqrt{A \cos \left(\frac{2 \pi}{L} x\right)+1} .
$$

A vanishing initial velocity field is assumed and thus $\Psi_{0}$ is real. In order to check temporal convergence, we vary the time step $\Delta t$ and consider the discrete $L_{2}$ error in the density contrast, i.e., $\epsilon=\|\delta(x, t)-\tilde{\delta}(x, t)\|_{2}$, where $\tilde{\delta}(x, t)$ denotes a reference solution computed on the finest temporal grid, $\Delta t=10^{-5}$ in this case. In the non-autonomous case, the integration is performed from $z=500$ to present time redshift $z=0$, which corresponds to an initial time $t=0$ and final time of $t=52$ in our dimensionless units. To assess the impact of an expanding spacetime on the scheme's convergence, we rerun the numerical experiment for $a(t)=0.01$ and 0.1 , and compute density contrasts at the same values of $t$. Figure 1 depicts the results.

Independently of whether a static or expanding background cosmology is considered, Figure 1 allows one to distinguish two error regimes: Larger step sizes are dominated by the temporal error entering via the splitting, Equation (23), and scale, as expected, $\propto \Delta t^{2}$. For smaller timesteps further convergence is stopped by the scheme's spatial error. We rerun the simulation for various $N$ and only found minor impacts on the final error for small $\Delta t$. This convergence locking is most likely caused by the discontinuity of the Poisson kernel $-1 / k^{2}$ at $k=0$ which gets only more resolved as we increase the number of modes representable on the grid, see [39]. As expected, for increasing nonlinear parameter $a$, the convergence plateau shifts to ever smaller values of time step sizes.

Comparing the static runs, panel A and B in Figure 1, with the dynamic background for $\Omega_{m 0}=$ 0.3 employed in panel $C$ of Figure 1, shows that allowing for an expanding spacetime does not alter the error behavior qualitatively and error magnitudes within the convergence plateau are still comparable to midsized, constant interaction strength simulations. This is to be expected as due to the dependence of the scale factor on our time parameter, see Appendix A, Equation (10) is essentially a perturbed free Schrödinger equation for most of the time in the sense that the Hamiltonian takes

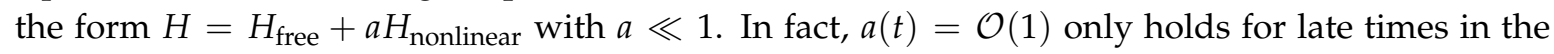
integration so that the impact of the nonlinearity on growing, unphysical error modes, i.e., their own gravitational collapse, is suppressed for most of the integration time. We conclude that the rather naïve approximation in Equation (22) suffices to follow the expansion of space adequately. 

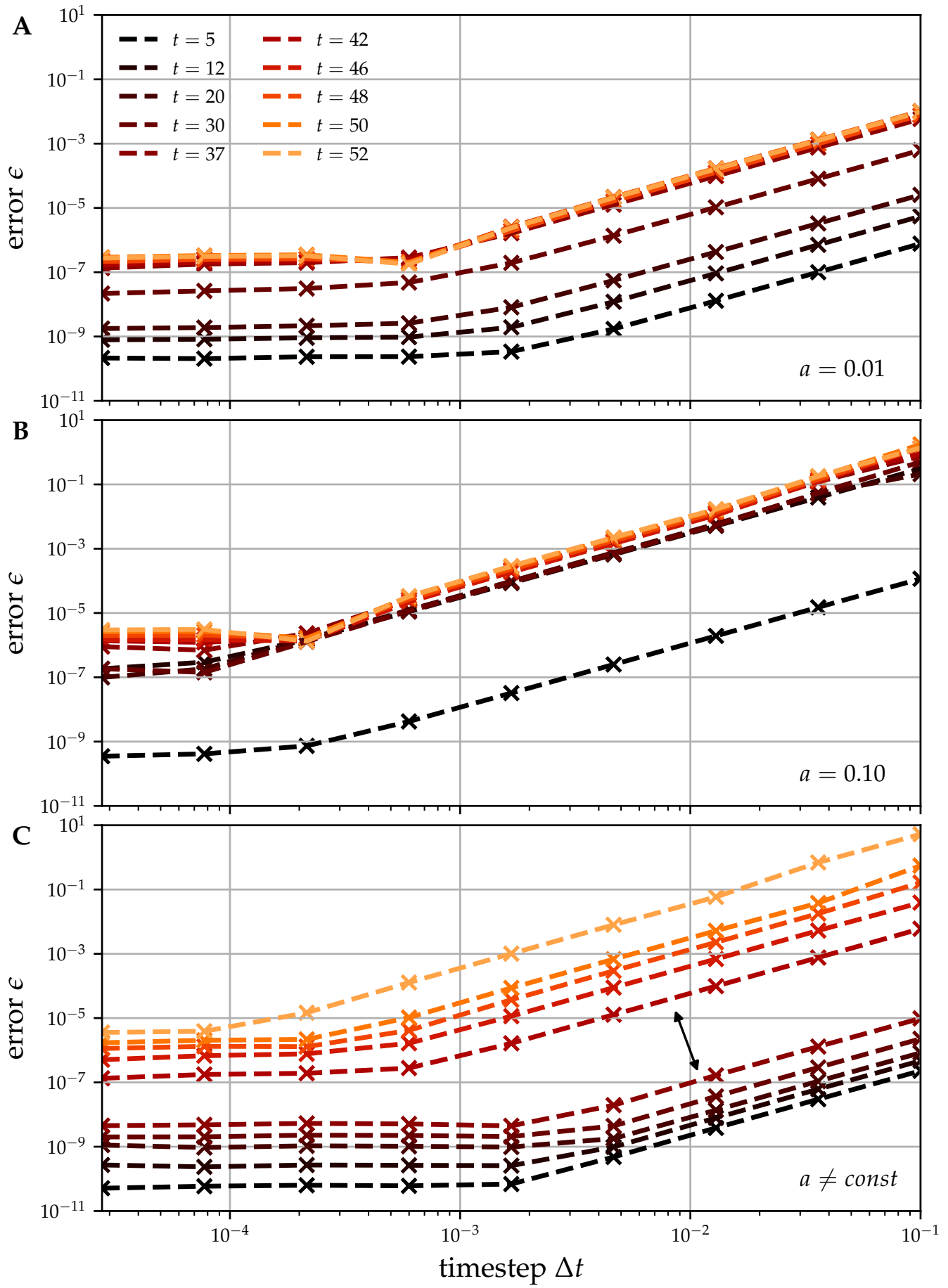

Figure 1. Convergence analysis for the collapse of a sine wave initial state, see Equation (25) with $N=16,384$ spatial points and various timestep sizes. The numerical solution is compared to a reference solution at coinciding values of $t$ as given in the legend. (A/B): Static spacetime with $a(t)=0.01, a(t)=0.1$. Evidently, for large $\Delta t$ the splitting error introduced by the time discretization in Equation (23) dominates and scales $\propto \Delta t^{2}$. For small $\Delta t$ convergence reaches a plateau in which the spatial discretization hinders further convergence. (C): Dynamic spacetime with $\Omega_{m 0}=0.3$. Again, two error regimes can be distinguished. Note that (i) as in the static spacetime case, the convergence plateau decreases in size as $a(t)$ approaches unity and (ii) the large gap indicated by the arrow between $z=50$ and $z=25$, i.e., $t=37$ and $t=42$. This is most likely caused by the first shell crossing event depicted in Figure 2B. 


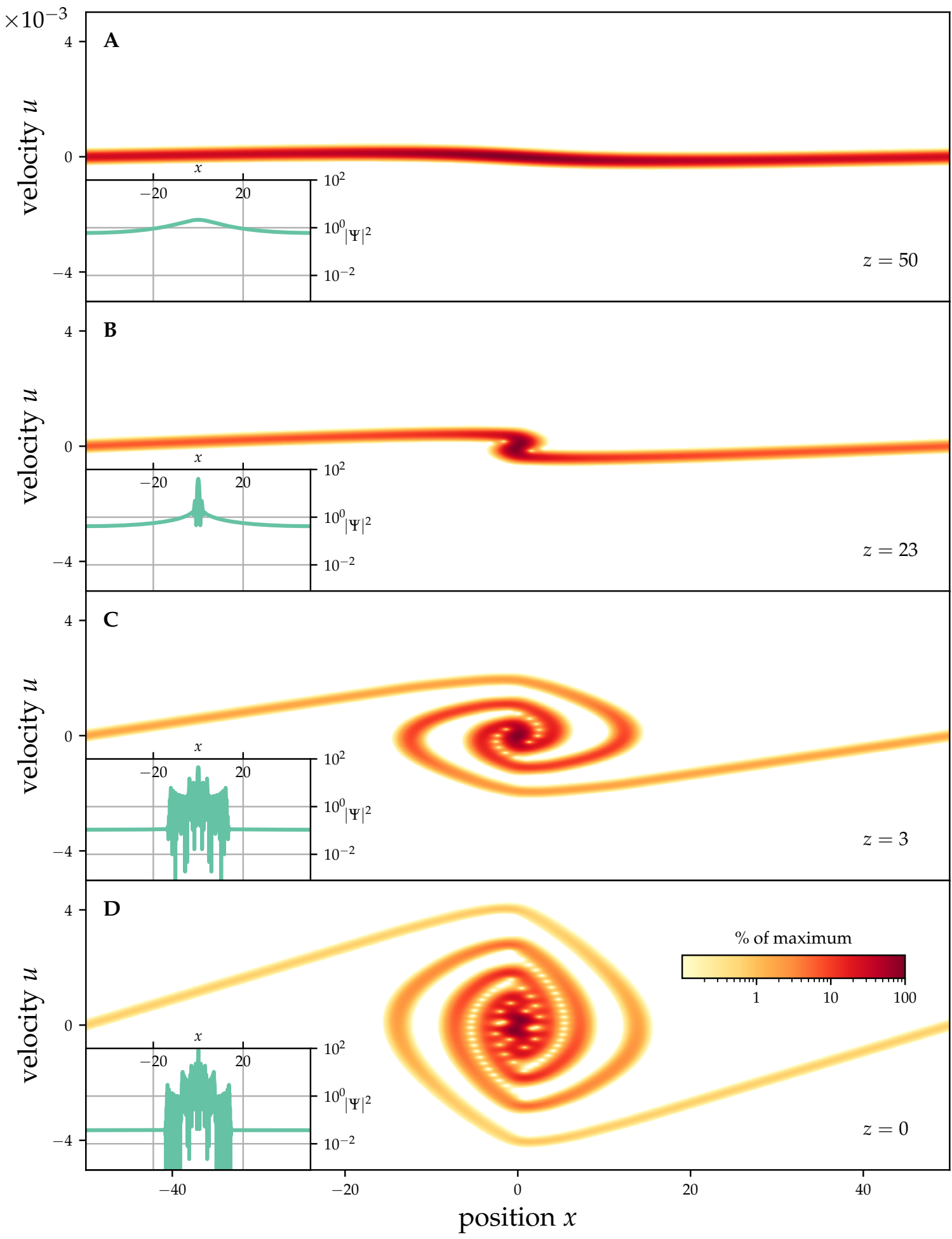

Figure 2. Gravitational collapse of a sinusoidal density perturbation in an expanding background cosmology with $\Omega_{m 0}=0.3$. Panel (A) depicts a snapshot in the single-stream regime which ends with a shell crossing shown in panel (B). Matter accumulation continues during the multi stream evolution in panel (C) and (D). Note that the collapse is not completed in panel D as matter still falls to the center. All distributions are normalized to their current maximum value. The insets illustrate the associated, unsmoothed density contrast on a logarithmic scale.

At last, we identify large error gaps in Figure 1, indicated as exemplary by the arrow in panel C, which appear earlier in the simulation if the interaction strength is large. We considered the associated 
density contrast and phase space distributions around the gaps and found that the first shell crossing, depicted in Figure 2B, is responsible for the sudden increase in the error $\epsilon$. Thus, although SPM has no problem in propagating the system through shell-crossings these singular events still pose a challenging numerical problem. Adjusting the stepsize dynamically might improve the numerical accuracy around these points in the evolution.

In what follows, we deem $\Delta t=10^{-4}$ and $N=16,384$ appropriate for our dynamic spacetime considerations. These values guarantee a stable integration in reasonable time of $\approx 5 \mathrm{~min}$ per run. Note that decreasing $\Delta t$ further does not improve accuracy due to the stalled convergence discussed above.

\section{Numerical Results}

\subsection{Phase Space Evolution of a Sinusoidal Perturbation}

To illustrate the prototypical stages a density perturbation undergoes in phase space, we study the evolution of a simple sine wave of the form $\delta\left(x, t_{\text {init }}\right)=-0.1 \cdot \cos \left(\frac{2 \pi}{L} x\right)$ discretized on $N=2^{14}$ equidistant grid points in an expanding background cosmology with $\Omega_{m 0}=0.3$. Using plane waves is a common choice for such studies as true cosmological initial conditions constitute a realisation of a Gaussian random field in $k$-space, i.e., upon retransformation into position space a superposition of sine waves with random but correlated amplitudes and phases. Moreover, the trivial, discrete spectrum makes it easy to precisely adjust the moment at which the gravitational collapse sets in. This is done via Jeans' stability criterion which is derived in [40] for SPM by linearizing Equation (8). In dimensionless form, the critical wavelength $\lambda_{J}$ given by this criterion reads:

$$
\lambda_{J}=\frac{2 \pi}{(6 a)^{1 / 4}} .
$$

Modes with $\lambda<\lambda_{J}$ are supported by the quantum pressure term in Equation (8) counteracting the collapse under self gravity mediated by the classical force term in Equation (8). The force balance changes in favour of gravity once the perturbation passes through the time dependent critical wavelength (26) initiating the inevitable collapse.

Heisenberg's relation for the space-velocity smoothing scales in Equation (4) reads $\sigma_{x} \sigma_{u}=\frac{1}{2}$ in our units. Thus, we set $\sigma_{x}=1 / \sqrt{2}$ in order to have the same resolution in both position and velocity space. Both box size and initial scale factor $a\left(t_{\text {init }}\right)$ satisfy the Jeans criterion. Starting from $z_{\text {init }}=500$, $L=100$ as chosen above is large enough such that the perturbation starts its gravitational collapse right at the onset of the integration. Figure 2 illustrates Husimi's distribution, see Equation (4), for four characteristic evolutionary stages: Figure 2A depicts the single stream situation in the linear growth regime of the density contrast. Here, matter slowly starts to fall into the gravitational well until both streams of matter meet at its origin and form a shell crossing in phase space, Figure 2B. At this point in time the distribution becomes perpendicular to the spatial axis and the classical dust model (see discussion around Equation (2)) breaks down. As noted before SPM is insensitive to such events and comfortably evolves the system forward into the multi stream regime shown in Figure 2C. Figure 2D depicts the situation at present time $z=0$, where matter of outer regions is still in free fall to the center of gravity.

From classical analysis one expects the approach of a dynamical equilibrium state. The latter is characterized by the fact that the viral theorem holds. We note that this equilibrium state is not a thermodynamic equilibrium, in the sense of maximal entropy, since such a state does not exist for gravitational systems of finite mass and energy, see Chapter 4 in [11].

If one seeks for a quantitative relaxation measure in the present quantum dynamical context Ehrenfest's theorem can be invoked to find the quantum analogue of the classical virial theorem, see $[13,41]$. In one dimension, one obtains:

$$
2(\langle T\rangle)_{\infty}=\left(\left\langle x \partial_{x} V\right\rangle\right)_{\infty}
$$


where $(\cdot)_{\infty}$ denotes time averages over infinite time.

Returning to our results in Figure 2: In a dynamical equilibrium state we would have expected a stationary core surrounded by a circularly shaped halo of matter in phase space. However, our phase space distribution in Figure 2D does not yet show a complete relaxation to an equilibrium state. Similar results are found in the literature: For an Einstein-de Sitter $\left(\Omega_{m 0}=1\right)$ universe employing the Schrödinger method see [14]; for the Vlasov counterpart see [30]; and finally for phase space collapse considerations using $\mathrm{N}$-Body techniques consider [28].

\subsection{Investigation of the Dynamical Equilibrium State}

To study the dynamical equilibrium state reached after collapse, we next consider Gaussian initial conditions for which matter is already close to the center of gravity. Doing so simplifies the numerical analysis since the free fall (collapse) time scale becomes shorter for spatially localized states. More specifically, we study the collapse in the core region for dynamic spacetime in Section 3.2.1. In the case of a static background cosmology, models for the asymptotic density profile are compared against our numerical simulations in Section 3.2.2.

\subsubsection{Core Region}

Taking all remaining parameters as outlined in Section 3.1 we obtain the present-time phase space distribution shown in Figure 3A. The initial condition was a Gaussian shaped density contrast with a standard deviation of $\sigma=8$, as illustrated in Figure 3A as black level lines in phase space (passing through the $1 \sigma$ and $2 \sigma$ in space). At $z=0$ the matter influx into the collapsed structure ceased and a dense but flat, cusp-free, core region embedded into a spatially fast decaying halo persists, see Figure 3B.

Interestingly, and contrary to results in $3+1$ dimensions [4,6-8] our results indicate a non-stationary, oscillating core region illustrated in the inset of Figure 3B. If we follow the evolution of the core region for a longer time no visible damping of these oscillations becomes apparent. If ever, the relaxation into a stable, stationary ground state occurs over time scales much larger than cosmologically and numerically justifiable. We interpret the attained localized, time periodic state as a "superposition" of a ground state soliton and excited nonlinear modes also known as breathers in the context of nonlinear systems, see e.g., [26]. In fact, carrying out a temporal Fourier analysis (not shown here) reveals a dominating 0 -mode, the ground state, together with three to five harmonics supporting our interpretation of a nonlinear superposition. We checked the existence of a stationary soliton in the $1+1$ case by means of an imaginary time propagation which filters out the true ground state for any initial condition that contains the former.

The numerical result that the mentioned superposition of nonlinear states does not decay into the stationary ground state on our time scales of interest indicates that energy transport, in particular transport of excess kinetic energy out of the potential well, often dubbed gravitational cooling $[4,13]$, is not occurring. We also checked whether the final state got "reheated" due to streams of matter reentering the domain as a consequence of our periodic boundary conditions but did not find a significant matter flux across the domain boundaries. The authors of [19] argue that the observed suppression of gravitational cooling is to expected in $1+1$ dimensions and suggest a modification of Poisson's equation (10) to stimulate energy transfer into unbound radiation modes of the modified potential. We leave a further investigation of this subject to a future work. 


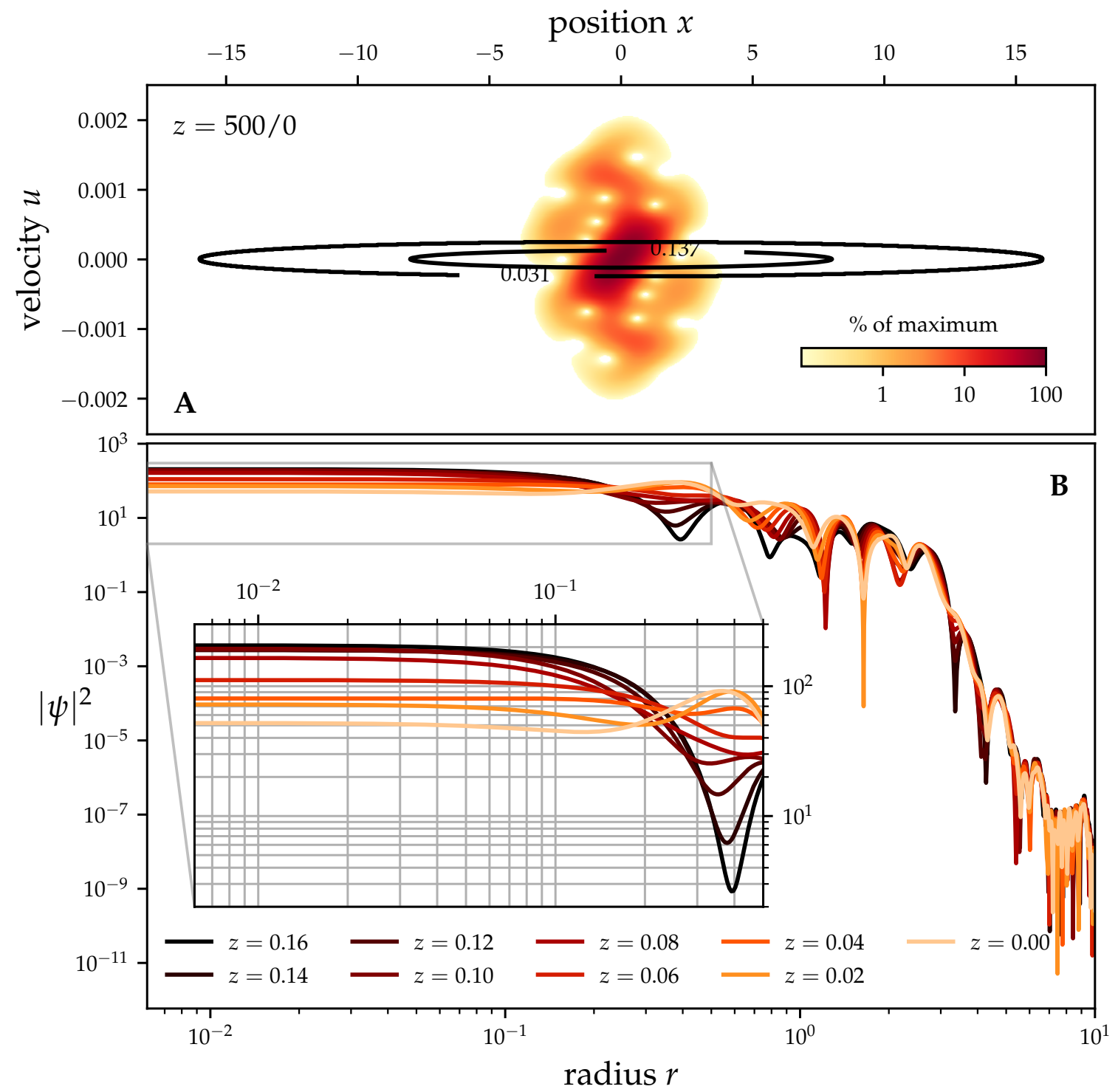

Figure 3. Gravitational collapse of a Gaussian density perturbation at $z=500$ up to present time. Parameters are chosen as in Section 3.1. (A) depicts initial (black level lines) and final state in phase space. Both densities are normalized to the maximum values of the final matter distribution. (B) illustrates the oscillatory behavior of the core region of the radial, i.e., positive coordinate half space, density $|\Psi|^{2}$ at late integration times.

\subsubsection{Halo Profile}

In this section, we consider whether the distribution of matter constituting the halo region of the collapsed structure recovers the classical CDM expectation. As mentioned before, in $3+1$ dimensions this means embedding a soliton core into a NFW profile that scales $\propto r^{-3}$. For the $1+1$ situation at hand and a static $a=1$ spacetime, [27] proposes a $\rho(r) \propto r^{-1 / 2}$ scaling. The authors of [28] combine the power-law assumption with an Einasto profile [42] that suppresses the density distribution exponentially:

$$
\rho(r) \propto r^{-\gamma} \exp \left(-\left(r / r_{0}\right)^{2-\gamma}\right), \quad r=|x|, \quad 0<\gamma<1 .
$$

We follow this argumentation and consider the same Gaussian initial condition as before but this time with $a=1$ throughout the entire integration time up to $t=250$. Figure 4A illustrates 
both initial and final density distribution $|\Psi|^{2}$. In fact, the entire distribution is strongly fluctuating, far more than in the dynamic case in Figure 3. Nevertheless, it keeps its overall shape. Therefore, we tacitly invoke the ergodic hypothesis and consider the time average of the distribution in the interval $t \in[249.5,250]$. Moreover, in accordance with [17], we integrate $\rho(r)$ and normalize the result to the total mass $M_{\text {tot }}$ inside the box. The reason for doing so is that good correspondence between SPM and Vlasov observables can in general only be expected after smoothing on spatial scales $\sigma_{x}$, see Section 1.2. Integrating in space spares us the necessity of choosing such a scale. Equation (28) becomes:

$$
\frac{M(r)}{M_{\mathrm{tot}}}=-\frac{r_{0}^{1-\gamma}}{M_{\mathrm{tot}}(2-\gamma)^{2}}\left[\Gamma\left(\frac{1}{\gamma-2}\right)+(2-\gamma) \Gamma\left(1+\frac{1}{\gamma-2},\left(\frac{r}{r_{0}}\right)^{2-\gamma}\right)\right]
$$

where $\Gamma(\cdot)$ and $\Gamma(\cdot, \cdot)$ denote the complete and incomplete gamma function. Figure 4B illustrates the fit result showing a reasonable accordance between model and data. The blue shaded area visualizes the $1 \sigma$ interval obtained by propagating the sample standard deviation from the averaging procedure through the mass normalization. We find an exponent of $\gamma \approx 0.5$ and check whether the result stays constant if the averaging window is altered.

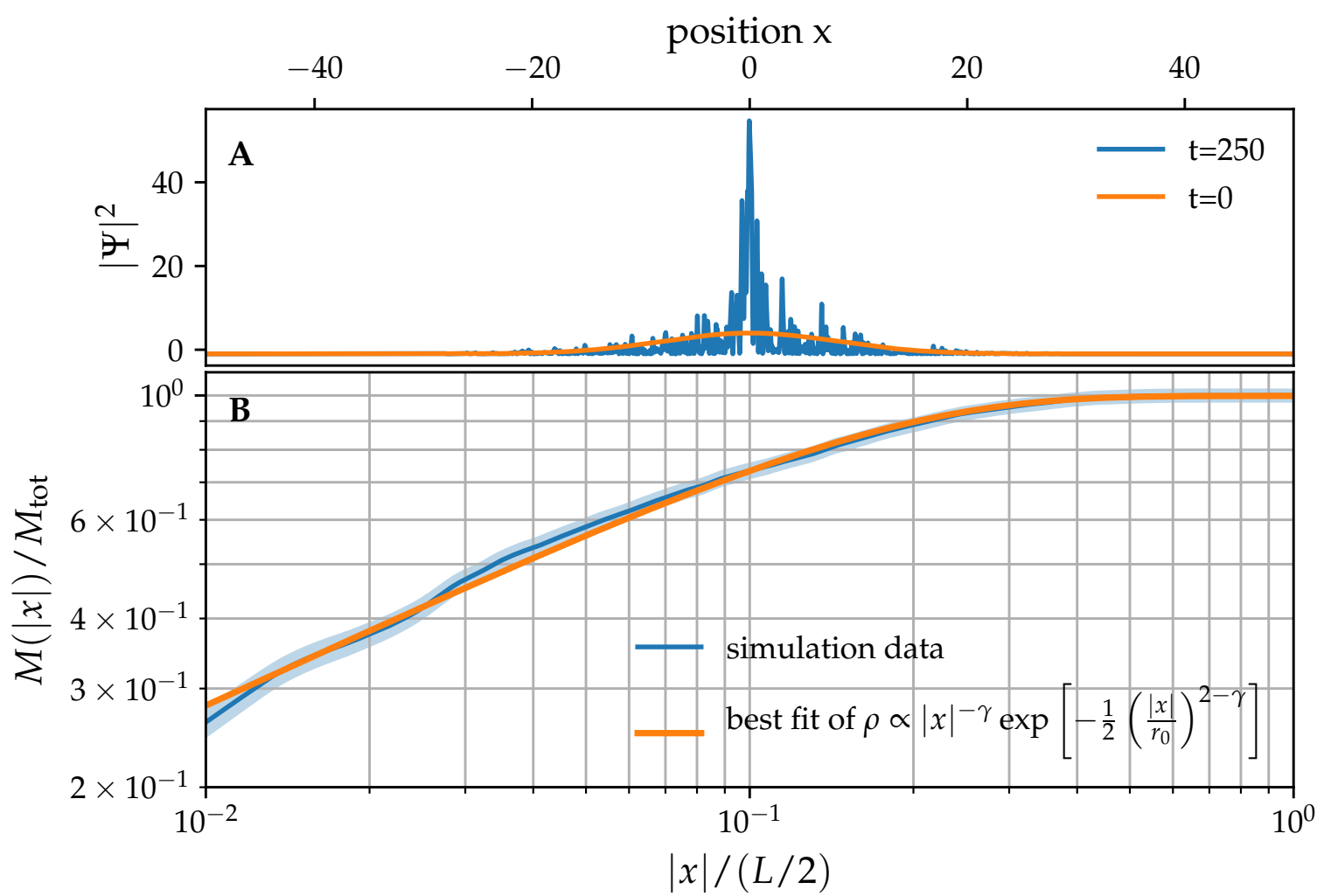

Figure 4. Collapse of Gaussian initial conditions in a static spacetime. (A) Comparison between initial (orange curve) and final state (blue curve). Note the asymmetry of the distribution with respect to the origin-an indication for non-negligible numerical errors. (B) Fit of the integrated, and time averaged halo with the classical model of Equation (29). The radial, cumulative mass distribution is obtained by averaging the positive and negative coordinate half space. Averaging was performed over 50 distributions equidistantly in the interval $t \in[249.5,250]$. The blue shaded region depicts the sample standard deviation after error propagation.

Note, however, that the quality of our data is by far not sufficient to decide on a concrete value $\gamma$ for multiple reasons. First, observe the unphysical asymmetry of the density distribution in Figure $4 \mathrm{~A}$ indicating that numerical errors play a non-negligible role for such long integration times. 
Second, we expect to approach the classical model in the semiclassical limit $\mu \rightarrow 0$. Changing $\mu$ in our conventions (9) means changing the adimensional box size which in turn by $\langle\delta\rangle=0$ has a direct impact on the initial conditions. Thus using a dimensionless form of Equation (3) which keeps $\mu$ as an independently adjustable parameter would be more appropriate for semiclassical considerations. This was done in [17] where the authors report good agreement with $\gamma=0.5$ as $\mu \rightarrow 0$.

\section{Conclusions and Prospects}

Our purpose was to illustrate key characteristics of the $3+1$ Schrödinger-Poisson model in $1+1$ dimensions. To this end, numerical studies for both spatially nonlocalized and localized initial conditions in static and dynamic background cosmologies were considered. Following the gravitational collapse by means of Husimi's distribution recovers the prototypical evolutionary stages in phase space. For sufficiently localized initial density perturbations dense cusp-free cores were found that oscillate periodically in time, whereas the core enclosing matter-the halo-follows a power-law scaling consistent with arguments for classical CDM.

Our results, in particular the characteristics of the dynamical equilibrium state, are qualitatively in accordance with results known from $d=3$ spatial dimensions. We stress that the existence of a solitonic density core, alleviating the core-cusp problem, is a common feature of the $d=1$ (this work), $d=2[31]$ and $d=3[6,8]$ dimensional problem irrespective of the cosmological background model. The oscillating behavior around this ground state solution is also found in two spatial dimensions, see [31], and we suspect comparable behavior for the $3+1$ case. Nevertheless, the reduced number of geometric degrees of freedom certainly affects the evolution of the scalar field especially in how fast the equilibrium state is attained. Recall the gravitational force of a point source derived from the solution of the $d$-dimensional Poisson equation scales as $F=-\nabla V \propto r^{-d+1}$. Consequently, for $d=1$, gravity is constant throughout the computational domain and matter is tightly bound to the collapsing structure rendering the ejection of excess mass and energy difficult. Furthermore, relaxation by means of the excitation of collective, phonon-like modes might be suppressed simply because in one dimension the timescale on which the gravitational potential changes is larger than the timescale set by the critical velocity of Landau's superfluid criterion [43]. At last, we note that in higher dimensions, the vortical degree of freedom plays an important role. In fact, quantized vortices are a common way of energy dissipation in a condensate driven by mechanisms like vortex reconnection and the classical Kolmogorov energy cascade [44].

The possibilities of extending this work are rather diverse. We remark on two major topics. Numerically, higher order, possibly adaptive, operator splitting methods should be considered and compared against the rather naïve splitting presented here. Moreover, other types of spatial discretizations might be competitive as well, especially in the light of the observed convergence locking in Section 2. These discretizations include basis expansions by means of Chebyshev polynomials [45] or localized B-splines [46]. From there, the next natural step is to extend our considerations into $2+1$ dimensions.

Although the $1+1$ case is somewhat artificial in a cosmological context, it nevertheless is a compelling physical system in its own right. In particular, we observed very long relaxation times in our simulation runs, mainly because the previously discussed gravitational cooling mechanisms seems to be suppressed in one spatial dimension. Understanding and controlling this phenomenon might deepen the comprehension of relaxation processes in higher dimensions as well.

Author Contributions: Project design: M.P. and S.W.; data production and analysis: T.Z.; all authors contributed to the interpretation of the results and to the writing of the manuscript.

Funding: Javier Madronero acknowledges the financial support from Vicerrectoría de Investigaciones (Univalle) grant number 71120 .

Conflicts of Interest: The authors declare no conflict of interest. 


\section{Appendix A. Cosmic Scalefactor in Code Time}

To construct the cosmic scale factor as a function of our dimensionless time parameter, we proceed as outlined in [30] and recast the time differential in Equation (9) to depend explicitly on the scale factor differential $\mathrm{d} a$. Let $H_{0}$ and $\Omega_{m 0}$ denote the present day Hubble constant and matter density parameter respectively. Then:

$$
\begin{aligned}
\mathrm{d} t & =\frac{1}{\dot{a} a^{2}}\left[\frac{3}{2} H_{0}^{2} \Omega_{m 0}\right]^{\frac{1}{2}} \mathrm{~d} a \\
& =\left[\frac{3 \Omega_{m 0}}{2\left(\Omega_{m 0} a^{-3}+\Omega_{\Lambda 0}\right)}\right]^{\frac{1}{2}} \mathrm{~d} a .
\end{aligned}
$$

The last equality is established by using the radiation free Friedman-Lemaitre equation, see [38]:

$$
H^{2}(a)=\left(\frac{\dot{a}}{a}\right)^{2}=H_{0}^{2}\left(\Omega_{m 0} a^{-3}+\Omega_{\Lambda 0}\right) .
$$

Here $\Omega_{\Lambda 0} \equiv 1-\Omega_{m 0}$. To obtain $a(t)$ we first compute the inverse relation $t(a)$ by integrating Equation (A3) and subsequently invert the result to arrive at the scale factor as a function of adimensional time $t$. Unfortunately, it is not possible to integrate (A3) for all possible models set by $\Omega_{m 0}$ in closed form. Therefore, we divide the interval $\left[a_{\text {init }}, a_{\text {end }}\right]$ into $K$ equidistant parts $\Delta a=\frac{a_{\text {end }}-a_{\text {init }}}{K}$ and perform the integration numerically by means of the midpoint method:

$$
t\left(a_{k}\right)=t\left(a_{k-1}\right)+\left.\frac{\mathrm{d} t}{\mathrm{~d} a}\right|_{a_{k-1}+\frac{1}{2} \Delta a} \cdot \Delta a, \quad t\left(a_{\text {init }}\right)=0 .
$$

If we seek values of $t$ in between two grid points, linear interpolation is performed. If the value $t\left(a^{\prime}\right)$ is known, then inverting $t(a)$ at $a^{\prime}$ is equivalent to finding the root to

$$
0=t(a)-t\left(a^{\prime}\right)
$$

This is a task easily accomplished by a simple bisection algorithm with an initial bounding interval of $\left[a_{\text {init }}, a_{\text {end }}\right]$. Figure A1 illustrates the scale factor growth for six different values of $\Omega_{m 0}$ and late simulation times for which discrepancies in the expansion history become apparent.

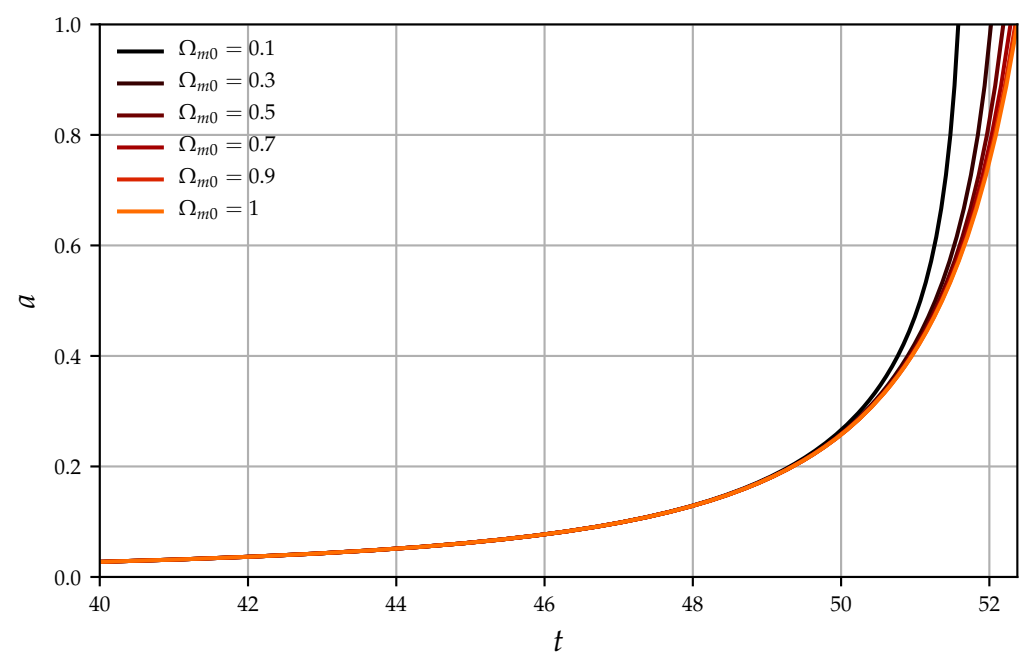

Figure A1. Cosmic scale factor as a function of adimensional time $t$ for $t>40$. The case $\Omega_{m 0}=1$ is known as the Einstein-de-Sitter model and constitutes a universe with zero dark energy. Having $\Omega_{\Lambda 0} \neq 0$ accelerates the expansion further. 


\section{References}

1. Boyd, R.W. Nonlinear Optics; Elsevier: Amsterdam, The Netherlands, 2008.

2. Dalfovo, F.; Giorgini, S.; Pitaevskii, L.P.; Stringari, S. Theory of Bose-Einstein condensation in trapped gases. Rev. Mod. Phys. 1999, 71, 463-512. [CrossRef]

3. Brian H.; Bransden, C.J.J. Physics of Atoms and Molecules; Prentice Hall: Harlow, UK, 2003.

4. Guzmán, F.S.; Ureña-López, L.A. Evolution of the Schrödinger-Newton system for a self-gravitating scalar field. Phys. Rev. D 2004, 69. [CrossRef]

5. Guzman, F.S.; Urena-Lopez, L.A. Gravitational Cooling of Self-gravitating Bose Condensates. Astrophys. J. 2006, 645, 814-819. [CrossRef]

6. Schive, H.Y.; Chiueh, T.; Broadhurst, T. Cosmic structure as the quantum interference of a coherent dark wave. Nat. Phys. 2014, 10, 496-499. [CrossRef]

7. Schwabe, B.; Niemeyer, J.C.; Engels, J.F. Simulations of solitonic core mergers in ultralight axion dark matter cosmologies. Phys. Rev. D 2016, 94. [CrossRef]

8. Mocz, P.; Vogelsberger, M.; Robles, V.H.; Zavala, J.; Boylan-Kolchin, M.; Fialkov, A.; Hernquist, L. Galaxy formation with BECDM - I. Turbulence and relaxation of idealized haloes. Mon. Not. R. Astron. Soc. 2017, 471, 4559-4570. [CrossRef]

9. Peebles, P.J.E. Large-Scale Structure of the Universe; Princeton University Press: Princeton, NJ, USA, 1980.

10. Collaboration, P. Planck 2018 Results. VI. Cosmological Parameters. arXiv 2019, arXiv:1807.06209v1

11. James Binney, T.S. Galactic Dynamics; Princeton University Press: Princeton, NJ, USA, 2008.

12. Widrow, L.M.; Kaiser, N. Using the Schroedinger Equation to Simulate Collisionless Matter. Astrophys. J. 1993, 416, L71. [CrossRef]

13. Hui, L.; Ostriker, J.P.; Tremaine, S.; Witten, E. Ultralight scalars as cosmological dark matter. Phys. Rev. D 2017, 95. [CrossRef]

14. Uhlemann, C.; Kopp, M.; Haugg, T. Schrödinger method as N-body double and UV completion of dust. Phys. Rev. D 2014, 90. [CrossRef]

15. Kopp, M.; Vattis, K.; Skordis, C. Solving the Vlasov equation in two spatial dimensions with the Schrödinger method. Phys. Rev. D 2017, 96. [CrossRef]

16. Mocz, P.; Lancaster, L.; Fialkov, A.; Becerra, F.; Chavanis, P.H. Schrödinger-Poisson-Vlasov-Poisson correspondence. Phys. Rev. D 2018, 97. [CrossRef]

17. Garny, M.; Konstandin, T. Gravitational collapse in the Schrödinger-Poisson system. J. Cosmol. Astropart. Phys. 2018, 2018, 009. [CrossRef]

18. Diósi, L. Gravitation and quantum-mechanical localization of macro-objects. Phys. Lett. A 1984, 105, $199-202$. [CrossRef]

19. Kaminer, I.; Rotschild, C.; Manela, O.; Segev, M. Periodic solitons in nonlocal nonlinear media. Opt. Lett. 2007, 32, 3209. [CrossRef]

20. Springel, V. The cosmological simulation code gadget-2. Mon. Not. R. Astron. Soc. 2005, 364, 1105-1134. [CrossRef]

21. Springel, V.; White, S.D.M.; Jenkins, A.; Frenk, C.S.; Yoshida, N.; Gao, L.; Navarro, J.; Thacker, R.; Croton, D.; Helly, J.; et al. Simulations of the formation, evolution and clustering of galaxies and quasars. Nature 2005, 435, 629-636. [CrossRef]

22. Bullock, J.S.; Boylan-Kolchin, M. Small-Scale Challenges to the $\Lambda$ CDM Paradigm. Annu. Rev. Astron. Astrophys. 2017. [CrossRef]

23. Schive, H.Y.; Liao, M.H.; Woo, T.P.; Wong, S.K.; Chiueh, T.; Broadhurst, T.; Hwang, W.Y.P. Understanding the Core-Halo Relation of Quantum Wave Dark Matter from 3D Simulations. Phys. Rev. Lett. 2014, 113. [CrossRef]

24. Madelung, E. Quantentheorie in hydrodynamischer Form. Z. Phys. 1927, 40, 322-326. [CrossRef]

25. Navarro, J.F.; Frenk, C.S.; White, S.D.M. The Structure of Cold Dark Matter Halos. Astrophys. J. 1996, $462,563$. [CrossRef]

26. Flach, S.; Willis, C. Discrete breathers. Phys. Rep. 1998, 295, 181-264. [CrossRef]

27. Binney, J. Discreteness effects in cosmological N-body simulations. Mon. Not. R. Astron. Soc. 2004, 350, 939-948. [CrossRef] 
28. Schulz, A.E.; Dehnen, W.; Jungman, G.; Tremaine, S. Gravitational collapse in one dimension. Mon. Not. R. Astron. Soc. 2013, 431, 49-62. [CrossRef]

29. Sousbie, T.; Colombi, S. ColDICE: A parallel Vlasov-Poisson solver using moving adaptive simplicial tessellation. J. Comput. Phys. 2016, 321, 644-697. [CrossRef]

30. Taruya, A.; Colombi, S. Post-collapse perturbation theory in 1D cosmology - beyond shell-crossing. Mon. Not. R. Astron. Soc. 2017, 470, 4858-4884. [CrossRef]

31. Navarrete, A.; Paredes, A.; Salgueiro, J.R.; Michinel, H. Spatial solitons in thermo-optical media from the nonlinear Schrödinger-Poisson equation and dark-matter analogs. Phys. Rev. A 2017, 95. [CrossRef]

32. Antoine, X.; Bao, W.; Besse, C. Computational methods for the dynamics of the nonlinear Schrödinger/Gross-Pitaevskii equations. Comput. Phys. Commun. 2013, 184, 2621-2633. [CrossRef]

33. Lubich, C. On splitting methods for Schrödinger-Poisson and cubic nonlinear Schrödinger equations. Math. Comput. 2008, 77, 2141-2153. [CrossRef]

34. Koch, O.; Neuhauser, C.; Thalhammer, M. Error analysis of high-order splitting methods for nonlinear evolutionary Schrödinger equations and application to the MCTDHF equations in electron dynamics. ESAIM: Math. Model. Numer. Anal. 2013, 47, 1265-1286. [CrossRef]

35. Blanes, S.; Casas, F. Splitting methods for non-autonomous separable dynamical systems. J. Phys. Math. Gen. 2006, 39, 5405-5423. [CrossRef]

36. Blanes, S.; Moan, P. Practical symplectic partitioned Runge-Kutta and Runge-Kutta-Nyström methods. J. Comput. Appl. Math. 2002, 142, 313-330. [CrossRef]

37. Blanes, S.; Casas, F.; Oteo, J.; Ros, J. The Magnus expansion and some of its applications. Phys. Rep. 2009, 470, 151-238. [CrossRef]

38. Peebles, P.J.E. Principles of Physical Cosmology; Princeton University Press: Princeton, NJ, USA, 1993

39. Bao, W.; Jiang, S.; Tang, Q.; Zhang, Y. Computing the ground state and dynamics of the nonlinear Schrödinger equation with nonlocal interactions via the nonuniform FFT. J. Comput. Phys. 2015, 296, 72-89. [CrossRef]

40. Woo, T.P.; Chiueh, T. High-Resolution Simulation on Structure Formation with Extremely Light Bosonic Matter. Astrophys. J. 2009, 697, 850-861. [CrossRef]

41. LeBohec, S. Quantum Mechanical Approaches to the Virial. Available online: https://pdfs.semanticscholar. org/5d67/14575ef5d5d091cca4b01b423712f25164e9.pdf (accessed on 6 October 2019)

42. Einasto, J. On the Construction of a Composite Model for the Galaxy and on the Determination of the System of Galactic Parameters. Tr. Astrofiz. Inst.-Alma-Ata 1965, 5, 87-100.

43. Lev. P. Pitaevskii, S.S. Bose-Einstein Condensation and Superfluidity; Oxford University Press: Oxford, UK, 2016.

44. Kobayashi, M.; Tsubota, M. Kolmogorov Spectrum of Superfluid Turbulence: Numerical Analysis of the Gross-Pitaevskii Equation with a Small-Scale Dissipation. Phys. Rev. Lett. 2005, 94. [CrossRef]

45. Chen, R.; Guo, H. The Chebyshev propagator for quantum systems. Comput. Phys. Commun. 1999, 119, $19-31$. [CrossRef]

46. Carl de Boor, C.D. A Practical Guide to Splines; Springer: Berlin/Heidelberg, Germany, 2001.

(C) 2019 by the authors. Licensee MDPI, Basel, Switzerland. This article is an open access article distributed under the terms and conditions of the Creative Commons Attribution (CC BY) license (http://creativecommons.org/licenses/by/4.0/). 